\title{
Prescribing Ricci curvature on open surfaces
}

\author{
Jianguo $\mathrm{CAO}^{1}$ and Dennis DeTURCK ${ }^{2}$ \\ Dedicated to Professor Noboru Tanaka on the occasion of his sixtieth birthday \\ (Received May 8, 1990, Revised August 6, 1990)
}

\section{Introduction.}

This work grew out of an attempt to understand the problem of finding a complete, rotationally symmetric metric on $\boldsymbol{R}^{n}$ for $n \geq 3$ with a prescribed, rotationally symmetric Ricci tensor. As will be reported elsewhere, one runs headlong into the problem of deciding whether a given "Ricci candidate" on a surface (in particular, on $\boldsymbol{R}^{2}$ ) is in fact the Ricci tensor of a complete metric on the surface. The issue of completeness is surprisingly delicate, even for surfaces, and so the subject of this paper is the following :

PROBLEM. Given a symmetric covariant tensor $R$ of rank two on an open surface $S$ (of finite topological type), when does there exist a complete metric $g$ such that $\operatorname{Ric}(g)=R$ on $S$ ?

Of course, by $\operatorname{Ric}(g)$, we mean the (covariant) Ricci tensor of the metric $g$. Once one has existence of such a metric, it becomes reasonable to ask to what extent the metric is unique (since $\operatorname{Ric}(c g)=\operatorname{Ric}(g)$ for any positive constant $c$, the best uniqueness statement possible is "unique up to a constant multiple"). As we shall see below (Lemma 1.4), it can be directly determined from the tensor $R$ whether the metric we seek will have finite or infinite total curvature. We give a fairly complete solution of the problem in the finite-total-curvature case (Theorem 2.5), including uniqueness statements, and we give sufficient conditions for the existence of a complete $g$ in the infinite-total-curvature case (Theorem 3.3). Examples 3.1 and 3.2 indicate why the issue of completeness in the latter case is so delicate.

On two-dimensional manifolds, one can take advantage of the fact that the Ricci tensor is equal to the Gauss curvature times the metric ten-

\footnotetext{
${ }^{1}$ Supported by NSF Grant DMS-8610730 at the Insititute for Advanced Study

${ }^{2}$ Supported by NSF Grant DMS-9001707 and the Institute for Advanced Study
} 
sor, i. e.,

(1.1) $\operatorname{Ric}(g)=K(g) \cdot g$.

As remarked in [D1], this gives the obvious necessary condition that in order for the symmetric two-tensor $R$ to be the Ricci tensor of a Riemannian metric, even locally, $R$ must be either positive definite, negative definite, or zero at each point. More precisely, there must exist a scalar function $x$ and a positive-definite tensor $\gamma$ such that

$$
R_{i j}=\varkappa \gamma_{i j}
$$

Given a tensor $R$ which satisfies (1.2), we see from equation (1.1) that the metric which satisfies $\operatorname{Ric}(g)=R$ must be pointwise conformal to $\gamma$, i. e., $g=e^{2 u} \gamma$ for a scalar function $u$ (unless $x \equiv 0$ on an open set). Furthermore, one has the following formula which relates the Ricci tensors of $g$ and $\gamma$ :

$$
\operatorname{Ric}(g)=\operatorname{Ric}(\gamma)+\left(\Delta_{\gamma} u\right) \gamma
$$

where $\Delta_{\gamma}$ is the Laplacian in the $\gamma$ metric (our sign convention would yield $\Delta u=-u^{\prime \prime}$ on $S^{1}$ ). Since the Laplace equation is always locally solvable, we recover the local existence result in [D1]:

Proposition 1.3. Given a tensor $R$ which satisfies (1.2) in a neighborhood of $p \in \boldsymbol{R}^{2}$, there is a metric $g$ defined in a neighborhood of $p$ such that $\operatorname{Ric}(g)=R$.

We remark that at points where $R \neq 0$, the metric $g$ will be at least as smooth as $R$.

To elicit global properties of $g$ from knowledge of $R$, we observe that two important two-forms are available from the information contained in $R$ alone. For any tensor $T$, we define $d V_{T}$ to be $\sqrt{\operatorname{det} T} d x d y$ in local coordinates, as long as det $T \geq 0$.

LEMMA 1.4. Suppose $\operatorname{Ric}(g)=R$ and $K$ is the Gauss curvature of $g$. Then, for any decomposition of $R$ of the form (1.2),

$$
\begin{aligned}
& \text { (i) } K d V_{g}=\varkappa d V_{\gamma} \\
& \text { (ii) }|K| d V_{g}=d V_{R}=|\chi| d V_{\gamma} .
\end{aligned}
$$

Since ( i ) is the Gauss-Bonnet integrand, we get a necessary (and sufficient!) condition for $R$ to be the Ricci curvature of a metric on a compact surface, namely that the integral of $x d V_{\gamma}$ be $2 \pi$ times the Euler characteristic (this was also reported in [D1]). On open surfaces, we will 
use both ( $\mathrm{i}$ ) and (ii) to help us decide whether a complete metric exists for a given Ricci candidate $R$. First, we give some examples to show that the situation can be somewhat complicated.

EXAMPLE 1.5. Let $R$ be the standard Euclidean metric on $\boldsymbol{R}^{2}$. Then for any $C^{\infty}$ function $f$, there exists a metric $g$ such that $\operatorname{Ric}(g)=f R$. To see this, we seek $g$ in the form $g=e^{2 u} R$, where $u$ must satisfy $\Delta u=f$. A Runge-theorem argument (see Corollary 3.5.2 of [Ho]) guarantees the existence of such a $u$. On the other hand, there is no complete metric $g$ on $\boldsymbol{R}^{2}$ with $\operatorname{Ric}(g)=R$. This follows from the classical inequality of Cohn-Vossen [CV], which says that if a complete surface has non-negative Gauss curvature, then

$$
\int K d V \leq 2 \pi
$$

Of course, for our choice of $R$, this integral is infinite.

EXAMPLE 1.6. With $R$ as in Example 1.5, we find that there are infinitely many nonisometric complete Riemannian metrics with Ricci tensor $-R$ on $\boldsymbol{R}^{2}$. In particular, using (1.3) with $\gamma=d x^{2}+d y^{2}$ on $\boldsymbol{R}^{2}$, we find that any metric of the form

$$
e^{\frac{1}{4}\left(x^{2}+y^{2}\right)+h}\left(d x^{2}+d y^{2}\right)
$$

will satisfy $\operatorname{Ric}(g)=-R$, where $h$ is any harmonic function on $\boldsymbol{R}^{2}$. Various choices of quadratic harmonic polynomials for $h$ (with leading coefficients greater than $-1 / 4)$ will provide complete metrics.

EXAMPLE 1.7. On $\boldsymbol{R}^{2}$, let

$$
R_{C}=\frac{C}{\left(1+r^{2}\right)^{2}}\left(d r^{2}+r^{2}\left(1+r^{2}\right) d \theta^{2}\right),
$$

where $r$ and $\theta$ are the usual polar coordinates on $\boldsymbol{R}^{2}$. It is an easy computation that

$$
\int_{R^{2}} d V_{R_{C}}=\int_{0}^{2 \pi} \int_{0}^{\infty} \frac{C r}{\left(1+r^{2}\right)^{3 / 2}} d r d \theta=2 \pi C,
$$

therefore, by Cohn-Vossen's inequality we can only hope to have a complete metric $g_{c}$ such that $\operatorname{Ric}\left(g_{c}\right)=R_{C}$ for $C \leq 1$. However, it will be a consequence of Theorem 2.5 that there is no complete metric on $\boldsymbol{R}^{2}$ with $\operatorname{Ric}(g)=R_{C}$ for any $C \neq 0$.

EXAMPLE 1.8. Let $f_{A}(r)$ be the characteristic function of the inter- 
val $[0, A]$, and set $R_{A}=f_{A}(r)\left(d r^{2}+r^{2} d \theta^{2}\right)$ on $\boldsymbol{R}^{2}$. Our solutions of $\operatorname{Ric}\left(g_{A}\right)$ $=R_{A}$ will be required to be $C^{1}$ across the circle $r=A$ (one could with more effort use a smooth function $f$, but ours is simpler and illustrates our point more easily). Cohn-Vossen's inequality applies again to give the restriction $A \leq \sqrt{2}$ if we are looking for complete metrics. Again, we find rotationally symmetric solutions of $\operatorname{Ric}\left(g_{A}\right)=R_{A}$, such that

$$
g_{A}=r^{-A^{2}}\left(d r^{2}+r^{2} d \theta^{2}\right)
$$

outside the disk where $r \leq A$. Note that our rotationally symmetric metrics $g_{A}$ are complete for all $A \leq \sqrt{2}$.

ExAmple 1.9. Let $S=\boldsymbol{R} \times S^{1}$ be a cylinder, and let $(t, \theta)$ be coordinates on the cylinder $(-\infty<t<\infty, 0 \leq \theta<2 \pi)$ so that $\gamma=d t^{2}+d \theta^{2}$ is the "standard" flat metric on $S$. As in Example 1.8, let $f_{A}(t)$ be the characteristic function of the interval $[0, A]$. Since $\Delta_{r}(u)=-\left(u_{t t}+u_{\theta \theta}\right)$, it is easy to see that there is no complete metric of the form $g=h(t) \gamma$ with $\operatorname{Ric}(g)=f_{A} \gamma$ for $A>0$. Of course, the metric $\gamma$ is itself complete (and flat), and is in fact the unique complete flat metric in its pointwise conformal class. Finally there are infinitely many nonisometric complete metrics such that $\operatorname{Ric}(g)=-f_{A} \gamma$ for any $A>0$.

\section{Finite total curvature.}

In this section, we use some theorems of Huber and Finn concerning the geometry of complete open surfaces to understand completely our problem in the case that the two-form in part (ii) of Lemma 1.4 has a finite integral. In other words, we will consider the problem of prescribing a tensor $R$ on our open surface $S$ such that

$$
\int_{S} d V_{R}<\infty .
$$

We begin our study by recalling some results about complete surfaces with finite total curvature.

Proposition 2.1. Let $(S, g)$ be a complete surface such that

$$
\int|K| d V_{g}<\infty .
$$

Then

(i) $S$ is homeomorphic to $\bar{S} \backslash\left\{p_{1}, \ldots, p_{n}\right\}$, where $\bar{S}$ is a compact surface and the $p_{i}$ are points of $\bar{S}$,

(ii) Each end of $S$ is parabolic, 
(iii) Each $p_{i}$ has a (punctured) neighborhood on which $g$ is isometric to the metric

$$
e^{2 u_{i}}|z|^{2 \beta_{i}}|d z|^{2}
$$

in terms of a local complex parameter on $\bar{S}$, where each $\beta_{i} \leq-1$, each $u_{i} \in L^{p}$ for all $1 \leq p<\infty$, and $\Delta u_{i} \in L^{1}$ (with respect to some smooth metric on $\bar{S}$ ),

(iv) The following modification of the Gauss-Bonnet formula holds :

$$
\frac{1}{2 \pi} \int_{s} K d V_{g}=\chi(\bar{S})+\sum_{i=1}^{n} \beta_{i}
$$

where the $\beta_{i}$ are as in (iii).

Part ( $\mathrm{i}$ ) is Theorem 13 and part (ii) is Theorem 15 in [H1]. Part (iii) is Satz 1 in [H2], and part (iv) is the main theorem of [F]. A resumé of these results also appears in [HT].

REMARKS. (i) The actual statement of Satz 1 in [H2] concerns a function $v$ defined on the exterior of a disk in $C$ such that $\Delta v \in L^{1}$ (finite total curvature for the metric $e^{2 v}|d z|^{2}$ ) and such that

$$
\int_{\Gamma} e^{v(z)}|d z|=\infty
$$

for any path $\Gamma$ diverging to infinity (completeness). The conclusion is a representation formula for $v$ as follows :

$$
v(z)=\frac{-1}{2 \pi} \iint_{|\zeta|>1} \log \left|1-\frac{z}{\zeta}\right| \Delta v(\zeta) d \xi d \eta+\beta \log |z|+h(z)
$$

where $\beta$ is a constant and $h$ is harmonic all the way out to infinity (in particular $h$ is bounded). One performs the inversion $z \mapsto 1 / z$ to pass to an equivalent formulation in a punctured disk, such that the metric $e^{2 v}|d z|^{2}$ has finite total curvature on the disk and any path which approaches the origin has infinite length:

$$
v(z)=\beta \log |z|+h(z)-\frac{1}{2 \pi} \iint_{0<|\xi|<1} \log |z-\zeta| \Delta v(\zeta) d \xi d \eta
$$

and $h(z)$ is harmonic in the whole unit disk (even at the origin). This immediately yields the representation (2.2) for the metric. The function $u$ in (2.2) is in $L^{p}$ for all $1 \leq p<\infty$ since it is the sum of a (locally bounded) harmonic function and the convolution of an $L^{1}$ function $(\Delta u)$ with the function $\log |z|$, which by Young's inequality is in $L^{p}$ for all $1 \leq p<\infty$. 
(ii) A metric with a representation of the form (2.2) near a parabolic end, will always be complete at that end provided $\beta_{i}<-1$ (and never if $\beta_{i}>-1$ ). If $\beta_{i}=-1$ then the metric may or may not be complete, as demonstrated by Example 1.8 above and Example 2.7 below. To show this for $\beta<-1$ (we drop the subscript $i$ for the time being), we use (2.2) and (2.4) to compute that the length of a radial curve $\Gamma$ in $0<|z|<1$ which "diverges" to the origin is $L=\int_{\Gamma} e^{u(z)}|z|^{\beta}|d z|$. If we apply Hölder's inequality to the integral $\int_{\Gamma}\left(|z|^{\beta / p} e^{u / p}\right) e^{-u / p}|d z|$, we get that

$$
L \geq \frac{\left(\int_{\Gamma}|z|^{\mid \beta / p}|d z|\right)^{p}}{\left(\int_{\Gamma} e^{-q u(z) / p}|d z|\right)^{p / q}} .
$$

We can choose $p>1$ so that the numerator on the right-hand side is infinite (since $\beta<-1$ ). As for the denominator, we first use (2.4) to write

$$
u(z)=h(z)-\frac{1}{2 \pi} \iint_{0<|\xi|<\varepsilon} \log |z-\zeta| \Delta u(\zeta) d \xi d \eta,
$$

where $h$ is harmonic (hence bounded, say $|h(z)|<M$ ) on $|z|<\varepsilon$. We decompose the measure $\Delta u(\zeta) d \xi d \eta$ into positive and negative parts (denoted $\left.d \mu^{ \pm}(\zeta)\right)$, we set

$$
u^{ \pm}(z)=\frac{-1}{2 \pi} \iint_{0<|\zeta|<\varepsilon} \log |z-\zeta| d \mu^{ \pm}(\zeta),
$$

and we set

$$
T^{ \pm}=\iint_{0<|\zeta|<\varepsilon}\left|d \mu^{ \pm}(\zeta)\right|
$$

We can now estimate the denominator as follows :

$$
\begin{aligned}
& \int_{\Gamma} e^{-q u(z) \mid p}|d z| \leq e^{M}\left\{\int_{\Gamma} \exp \left[\frac{-q}{2 \pi p} \iint_{0<|z|<\varepsilon} \log |z-\zeta| d \mu^{+}(\zeta)\right]|d z|+\right. \\
&\left.+\int_{\Gamma} \exp \left[\frac{-q}{2 \pi p} \iint_{0<|z|<\varepsilon} \log |z-\zeta| d \mu^{-}(\zeta)\right]|d z|\right\} \\
&=e^{M}\left\{\int_{\Gamma} \exp \left[\frac{-q T^{+}}{2 \pi p} \iint_{0<|z|<\varepsilon} \log |z-\zeta| \frac{d \mu^{+}(\zeta)}{T^{+}}\right]|d z|+\right. \\
&\left.+\int_{\Gamma} \exp \left[\frac{q T^{-}}{2 \pi p} \iint_{0<|z|<\varepsilon} \log |z-\zeta| \frac{\left|d \mu^{-}(\zeta)\right|}{T^{-}}\right]|d z|\right\} .
\end{aligned}
$$

We can use Jensen's inequality on the two integrals over $0<|z|<\varepsilon$ to obtain : 


$$
\begin{aligned}
\int_{\Gamma} e^{-q u(z) / p}|d z| \leq e^{M}\{ & \iiint_{\Gamma<|\zeta|<\varepsilon}|z-\zeta|^{-q T^{+/ /(2 \pi p)}} \frac{d \mu^{+}(\zeta)}{T^{+}}|d z|+ \\
& \left.+\int_{\Gamma} \iint_{0<|\zeta|<\varepsilon}|z-\zeta|^{q T^{-/}(2 \pi p)} \frac{\left|d \mu^{-}(\zeta)\right|}{T^{-}}|d z|\right\} .
\end{aligned}
$$

These integrals will be finite for any radial $\Gamma$ provided $\varepsilon$ is chosen small enough so that $\frac{-q T^{+}}{2 \pi p}>-1$. The proof that the metric is incomplete for $\beta>-1$ is similar (see [HT]; the ideas for these proofs originate in [R]).

(iii) Recall that if each end of the complete surface $S$ is parabolic, then $S$ admits no nonconstant bounded harmonic functions. This portends the uniqueness statements we will make for metrics on finite-totalcurvature surfaces.

From Proposition 2.1(iii), we see that, given a complete metric $g$ on a surface $S$ with finite total curvature, there is a smooth metric $g_{0}$ on the compact surface $\bar{S}$ such that $g=\rho g_{0}$, where $\rho$ is smooth except at the points $\left\{p_{1}, \ldots, p_{n}\right\}$ of $\bar{S} \backslash S$, and $\rho$ has singularities as indicated in (2.2) at the $p_{i}$. For ease of presentation in what follows, we make the following definition: For any function (distribution) $f$ on $S$ (or on $\bar{S}$ ), we define the function $\operatorname{reg}[f]$ on $\bar{S}$ by

$$
\operatorname{reg}[f](p)= \begin{cases}f(p) & \text { if } p \notin\left\{p_{1}, \ldots, p_{n}\right\} \\ 0 & \text { if } p \in\left\{p_{1}, \ldots, p_{n}\right\}\end{cases}
$$

The Ricci tensor of $g$ is of the form $x g_{0}$, where $x$ is smooth on $S$ but could have singularities at the $p_{i}$ with the restriction that $\operatorname{reg}[\chi]$ is $L^{1}$ on $\bar{S}$ with respect to $g_{0}$. This gives another necessary condition which a tensor $R$ (with finite $\left.\int d V_{R}\right)$ must satisfy in order to be the Ricci tensor of a complete metric on the open surface $S$ - namely, there must exist a smooth metric $g_{0}$ on the compactification $\bar{S}$ (obtained by adding a single point at each end), and an $L^{1}$ function $\operatorname{reg}[x]$ on $\bar{S}$ such that $R=x g_{0}$.

The Gauss-Bonnet formula (2.3) places even more restrictions on $R$. From Lemma 1.4, we can compute the integral on the left side of (2.3), in fact, this integral is equal to $\int \operatorname{reg}[\varkappa] d V_{g_{0}}$, where $x$ and $g_{0}$ are as in the previous paragraph. Of course, we know the Euler characteristic of $\bar{S}$, and we arrive at the following:

THEOREM 2.5. Let the (smooth) tensor $R$ be given on the open sur. face $S$, such that 


$$
\int d V_{R}<\infty
$$

If $R$ is the Ricci curvature of a complete metric on $S$, then

(i) $S$ is homeomorphic to $\bar{S} \backslash\left\{p_{1}, \ldots, p_{n}\right\}$, where $\bar{S}$ is a compact manifold without boundary.

(ii) $R$ can be realized as $R=x g_{0}$, where $g_{0}$ is a smooth metric on $\bar{S}$, $\operatorname{reg}[x] \in L^{1}\left(\bar{S}, g_{0}\right)$, and $x$ is smooth except at the $p_{i}$.

(iii)

$$
\frac{1}{2 \pi} \int_{s} \operatorname{reg}[\chi] d V_{g_{0}}-\chi(\bar{S}) \leq-n .
$$

Conversely, for each assignment of $\left\{\beta_{1}, \ldots, \beta_{n}\right\}$ such that each $\beta_{i} \leq-1$ and

$$
\sum_{i=1}^{n} \beta_{i}=\frac{1}{2 \pi} \int_{s} \chi d V_{g_{0}}-\chi(\bar{S})
$$

there is a unique (up to constant multiple) metric $g$ such that $\operatorname{Ric}(g)=R$ and $g$ is locally of the form (2.2) near $p_{i}$. If all the $\beta_{i}$ are chosen strictly less than -1 , then $g$ is guaranteed to be complete. If some $\beta_{i}=-1$, then the metric may or may not be complete at $p_{i}$.

The only statement which needs proving is the existence of $g$, given an appropriate assignment of the $\beta_{i}$. For this purpose, choose a metric $g_{1}$ $=e^{2 \phi} g_{0}$ on $S$ such that $g_{1}$ is of the form (2.2) at the $p_{i}$ with $\operatorname{reg}\left[\Delta_{g_{0}} \phi\right] \in$ $L^{1}\left(\bar{S}, g_{0}\right)$ (this is no problem, since (2.2) gives a recipe for $\phi$ near each $p_{i}$, and the metrics on the neighborhoods of the $p_{i}$ can then be smoothly patched together). By (1.3) and Proposition 2.1 we get that

$$
\operatorname{Ric}\left(g_{1}\right)=\left(K_{0}+\Delta_{g_{0}} \phi\right) g_{0},
$$

and $\operatorname{reg}\left[K_{0}+\Delta_{g_{0}} \phi\right]$ is in $L^{1}\left(\bar{S}, g_{0}\right)$. Note that

$$
\frac{1}{2 \pi} \int_{S}\left(K_{0}+\Delta_{g_{0}} \phi\right) d V_{g_{0}}=\chi(\bar{S})+\sum_{i=1}^{n} \beta_{i} .
$$

Moreover the function $\operatorname{reg}\left[\chi-\left(K_{0}+\Delta_{g_{0}} \phi\right)\right]$ is in $L^{1}\left(\bar{S}, g_{0}\right)$ and

$$
\int_{\bar{S}} \operatorname{reg}\left[x-\left(K_{0}+\Delta_{g_{0}} \phi\right)\right] d V_{g_{0}}=0 .
$$

Thus, there exists a function $v \in L^{1}\left(\bar{S}, g_{0}\right)$, unique up to an additive constant, such that $\Delta_{g_{0}} v=\operatorname{reg}\left[\chi-\left(K_{0}+\Delta_{g_{0}} \phi\right)\right]$. Again using (1.3) and Proposition 2.1, we see that the metric $g=e^{2 v} g_{1}$ is the metric we seek, and it is 
unique up to a multiplicative constant.

REMARKS. (i) The particular case of $\boldsymbol{R}^{2}$ (Examples 1.5 through 1. 8) becomes clearer now : since $\boldsymbol{R}^{2}=S^{2} \backslash\{p\}$, we see that we must have

$$
\frac{1}{2 \pi} \int_{R^{2}} d V_{R}-\chi\left(S^{2}\right)=\frac{1}{2 \pi} \int_{R^{2}} d V_{R}-2 \leq-1,
$$

which is the same as Cohn-Vossen's inequality. Moreover, we see that, since there is only one point in $S^{2} \backslash \boldsymbol{R}^{2}$, there is only one way to prescribe $\beta$, and so there can only be one complete solution of $\operatorname{Ric}(g)=R$ up to a multiplicative constant. In particular, when $\beta=-1$, the unique solution may be complete, as in Example 1.8 for $A=\sqrt{2}$, or it might not be complete as in Example 2.7 below, in which case there is simply no complete solution.

(ii) In Example 1.7, note that for $C \neq 0$, the tensor $R_{C}$ is a nonzero multiple of the complete metric $g_{0}=d r^{2}+r^{2}\left(1+r^{2}\right) d \theta^{2}$, and $g_{0}$ has a hyperbolic end (since $\int d r / r \sqrt{1+r^{2}}<\infty$, see $[M]$ ). The existence of a complete solution of $\operatorname{Ric}(g)=R_{C}$ would contradict Proposition 2.1( ii) and the conformal invariance of hyperbolicity.

(iii) Example 1.9 should also be clearer now. There are two points in $S^{2} \backslash S$, and since $\frac{1}{2 \pi} \int d V_{R}=-A$, we see that any choice of $\beta_{1}$ and $\beta_{2}$ which satisfies $\beta_{1}+\beta_{2}=-(2+A)$ with $\beta_{1} \leq-1$ and $\beta_{2} \leq-1$ will give rise to a complete metric (unique up to constant multiple) such that $\operatorname{Ric}(g)=f_{A} \gamma$.

EXAmPLE 2.7. For $\alpha>0$, let $R_{\alpha}$ be the tensor which is equal to

$$
R_{\alpha}=\frac{2(\alpha+2)}{e^{2}}\left(d r^{2}+r^{2} d \theta^{2}\right)
$$

for $r \leq e$, and

$$
R_{\alpha}=\frac{-2}{r^{2}(\log r)^{2}}\left(d r^{2}+r^{2} d \theta^{2}\right)
$$

for $r>e$, where $r$ and $\theta$ are the usual polar coordinates on $\boldsymbol{R}^{2}$. Since $R_{\alpha}$ is conformal to the Euclidean metric on $\boldsymbol{R}^{2}$, the end is parabolic. Moreover, we compute that

$$
\int_{R^{2}} d V_{R_{\alpha}}=2 \pi\left(\int_{0}^{e} \frac{2(\alpha+2)}{e^{2}} r d r+\int_{e}^{\infty} \frac{2}{r(\log r)^{2}} d r\right)=2 \pi(\alpha+4)<\infty,
$$

and, if $\operatorname{Ric}(g)=R_{\alpha}$, we would have 


$$
\int_{R^{2}} K_{g} d V_{g}=2 \pi\left(\int_{0}^{e} \frac{2(\alpha+2)}{e^{2}} r d r-\int_{e}^{\infty} \frac{2}{r(\log r)^{2}} d r\right)=2 \pi \alpha
$$

from Lemma 1.4. Therefore, Theorem 2.5 guarantees us a unique (up to constant multiple) metric $g_{\alpha}$ with $\operatorname{Ric}\left(g_{\alpha}\right)=R_{\alpha}$ (if we insist that $g_{\alpha}$ be $C^{1}$ across the circle $r=e$ where $R_{\alpha}$ is discontinuous). We find that this metric is given by

$$
g_{\alpha}=\exp \left(2-\alpha-\frac{\alpha+2}{e^{2}} r^{2}\right)\left(d r^{2}+r^{2} d \theta^{2}\right)
$$

for $r \leq e$, and

$$
g_{\alpha}=\frac{1}{r^{2 \alpha}(\log r)^{4}}\left(d r^{2}+r^{2} d \theta^{2}\right)
$$

if $r \geq e$. Note that $g_{\alpha}$ is complete if $\alpha<1$ but is incomplete if $\alpha \geq 1$, in accord with Cohn-Vossen's inequality, but in contrast to Example 1. 8.

\section{Infinite total curvature.}

In this section, we examine the problem of prescribing Ricci curvature when

$$
\int_{S} d V_{R}=\infty
$$

It was surprising to us that the issue of completeness of the solution of $\operatorname{Ric}(g)=R$ should be so delicate in this case (even if $R$ is assumed to be strictly negative definite on $S$ ).

EXAMPLE 3.1. One might be tempted to conjecture that, given any strictly positive function $f$ on $\boldsymbol{R}^{2}$, there would exist a complete metric $g$ whose Ricci tensor is equal to

$$
R=-f(x, y)\left(d x^{2}+d y^{2}\right)
$$

From our previous analyses, we know that such a metric would be of the form

$$
g=e^{2 u}\left(d x^{2}+d y^{2}\right)
$$

with $\Delta u=-f$. For completeness, we need that $u$ does not approach $-\infty$ too fast in any direction. A sufficient condition for completeness therefore would be for $u$ to be bounded from below. But the positivity of $f$ is not sufficient to guarantee the existence of a $u$ which is bounded from below, as the following example (kindly pointed out to us by E. Calabi) shows :

An elementary computation shows that if 


$$
u_{n}(z)=a_{n} \log \left(\varepsilon_{n}+\left|1-\frac{z}{n}\right|^{2}\right)
$$

then

$$
\Delta u_{n}(z)=\frac{-4 n^{2} \varepsilon_{n} a_{n}}{\left(n^{2} \varepsilon_{n}+|n-z|^{2}\right)^{2}}
$$

which is clearly negative, provided $a_{n}$ and $\varepsilon_{n}$ are positive. Furthermore,

$$
\iint_{R^{2}} \Delta u_{n}(z) d x d y=-4 \pi a_{n}
$$

We consider a function $u(z)$ of the form $u=\sum u_{n}$. In order for the series to converge uniformly on compact sets, we require $\varepsilon_{n} \rightarrow 0$ faster than $1 / n^{3}$, and $a_{n} \rightarrow 0$ at least as fast as $1 / n$. In fact, let us choose $a_{n}=1 / n$ and $\varepsilon_{n}=$ $e^{-e^{n}}$ (note that we then have $\int \Delta u=-\infty$ ). On the ball $B_{R}$ of radius $R$ centered at 0 , we can easily estimate $u(z)<40 R+10(\log R)^{2}$, but note that if $N$ is any positive integer, then $u_{N}(N)=-e^{N} / N$, and so $u(N)<-\frac{1}{2} e^{N} / N$ if $N$ is sufficiently large. We claim that there is no harmonic function $h(z)$ such that $u(z)+h(z)>0$ for all $z \in \boldsymbol{R}^{2}$. For suppose such an $h$ existed. Then on the ball of radius $2 N$, we would need $h(z)>-80 N-10(\log$ $2 N)^{2}$ in order for $u+h$ to be positive. Therefore, the function $h_{1}(z)=h(z)$ $+80 N+10(\log 2 N)^{2}$ is positive and harmonic on $B_{2 N}$. But the classical Harnack inequality [PW, page 108] says that $h_{1}(N)<3 h_{1}(0)$, in other words,

$$
h(N)<3 h(0)+160 N+20(\log 2 N)^{2} .
$$

Since $h(N) \geq \frac{1}{2} e^{N} / N$, this is a blatant contradiction for $N$ sufficiently large.

EXAMPLE 3.2. On the other hand, it is easy to see that if $f$ is any positive radial function on $\boldsymbol{R}^{2}$, then there is a (radial) complete metric satisfying $\operatorname{Ric}(g)=-f\left(d x^{2}+d y^{2}\right)$. One need only integrate the appropriate ordinary differential equation (the radial version of $\Delta u=-f$ ) with $u(0)=$ $u^{\prime}(0)=0$ to obtain the solution.

From the preceding examples, we conclude that some hypotheses on $R$ must be made in order to guarantee existence of a complete metric on $S$ satisfying $\operatorname{Ric}(g)=R$. These are summarized in the following theorem.

THEOREM 3.3. Let $S$ be an open surface of finite topological type and let $R$ be a tensor on $S$ with 


$$
\int d V_{R}=\infty
$$

Assume that $R$ can be expressed as $\phi g_{0}$, where $\phi$ is a smooth function on $S$ and $g_{0}$ is a complete metric on $S$ (this is a necessary condition for existence). With the conformal sturcture induced by $g_{0}, S$ will have three kinds of ends :

(i) Parabolic ends about which $\int d V_{R}<\infty$

(ii) Parabolic ends about which $\int d V_{R}=\infty$

(iii) Hyperbolic ends (about which $\int d V_{R}=\infty$ - this is again a necessary condition, see Proposition 2.1( ii )).

Let $\bar{S}$ be the compactification of $S$ obtained by adding points $\left\{p_{1}, \ldots, p_{m}\right\}$ at the parabolic ends and closed disks $\left\{D_{m+1}, \ldots, D_{n}\right\}$ at the hyperbolic ends, and so that $g_{0}=x g_{1}$, where $g_{1}$ is a smooth metric on the compact surface $\bar{S}$ and $x$ is smooth on $S$. About each $p_{i}$ and $D_{i}$, choose a neighborhood $U_{i}$ so that $U_{i} \cap U_{j}=\emptyset$ if $i \neq j$ and so that $g_{1}$ is uniformized by the complex parameter $z_{i}$ on $U_{i}$. For all the ends of types (ii) and (iii), assume that $x \phi$ can be decomposed as a sum of two functions $x \phi=r_{i}+b_{i}$ on $U_{i} \backslash\left\{p_{i}\right\}$ (resp. $\left.U_{i} \backslash\left\{D_{i}\right\}\right)$ with $r_{i}$ smooth, nonnegative, radial (i.e., a function of $\left|z_{i}\right|$ alone) and compactly supported in $U_{i}$, and so that

$$
\int_{U_{i}}\left|b_{i}\right| d V_{g_{1}}<\infty \text {. }
$$

Then there exists a complete metric (in fact, many complete metrics) $g$ such that $\operatorname{Ric}(g)=R$ on $S$.

REMARKS. (i) Despite appearances, the conditions in the theorem are independent of the choice of $g_{0}$ and $g_{1}$ within their conformal class. Of course, the existence of $g_{1}$ is guaranteed by the classical uniformization theorem of Poincaré and Koebe.

(ii) Note that the Ricci candidate of Example 3.1 suffers precisely from being too wildly nonradial near the end of $\boldsymbol{R}^{2}$. The last condition in the theorem is meant precisely to control such behavior.

PROOF. We first prove the theorem assuming that all the ends are parabolic (i. e., of types (i) and (ii)). Let $I=\left\{i \mid \int_{U_{i}} d V_{R}=\infty\right\}$ and $F=$ $\left\{i \mid \int_{U_{i}} d V_{R}<\infty\right\}$. Since the total curvature is infinite, $I$ is nonempty. 
Moreover, by the hypotheses on the behavior of $R$ near the $p_{i}$ for $i \in I$, we see that the measure

$$
d \mu=d V_{R}-\sum_{i \in I} r_{i} d V_{g_{1}}
$$

is integrable on $S$, and $\int_{S} d \mu<\infty$. For each $i \in F$, choose $\beta_{i}<-1$ (we could let $\beta_{i}=-1$, but the resulting metric might not be complete at $p_{i}$ ), and specify a function $\tau_{i}$ on $S$ which equals $\beta_{i} \log \left|z_{i}\right|$ in some neighborhood $V_{i} \subset U_{i}$ and is $\equiv 0$ outside $U_{i}$. By adjusting one or more of the $r_{i}$ 's (for $i \in I$ ) by a radial, $g_{1}$-integrable function, we can arrange that

$$
\int_{\bar{S}} \operatorname{reg}\left[d \mu-\left(K_{1}+\Delta_{g_{1}} \sum_{i \in F} \tau_{i}\right) d V_{g_{1}}\right]=0
$$

where $K_{1}$ is the Gauss curvature of $g_{1}$. Thus, there exists a function $u$, smooth on $S$, such that

$$
\left(\Delta_{g_{1}} u\right) d V_{g_{1}}=d \mu-\left(K_{1}+\Delta_{g_{1}} \sum_{i \in F} \tau_{i}\right) d V_{g_{1}}
$$

on $S$, i. e.,

$$
\Delta_{g_{1}} u=\varkappa \phi-\sum_{i \in I} r_{i}-K_{1}-\Delta_{g_{1}} \sum_{i \in F} \tau_{i}
$$

Also, by solving ordinary differential equations, we obtain a (unique) function $v$ such that $v \equiv 0$ on $S \backslash \cup_{i \in I} U_{i}$ and such that $v$ is radial on $U_{i}$ with $\Delta_{g_{1}} v=r_{i}$ on $U_{i}$. The upshot of all this is the equation

$$
\Delta_{g_{1}}\left(u+v+\sum_{i \in F} \tau_{i}\right)=\chi \phi-K_{1}
$$

which implies

$$
\operatorname{Ric}\left(e^{2\left(u+v+\Sigma \tau_{i}\right)} g_{1}\right)=R,
$$

and the metric (and hence the proof in this case) is complete.

It is no harder to deal with hyperbolic ends. When the Ricci candidate $R$ is decomposed as $\phi g_{0}$, with $g_{0}$ complete on $S$, we need only fill in the hyperbolic ends with disks rather than points to obtain the conformal completion $\bar{S}$ of $S$ (by the uniformization theorem again). We adjust the definition of $\operatorname{reg}[f]$ accordingly, by defining $\operatorname{reg}[f]$ to be zero throughout the added disks. One again obtains a smooth metric $g_{1}$ on $\bar{S}$ with $g_{0}=x g_{1}$ on $S$, and the assumptions about $x \phi$ near the hyperbolic ends (decomposition of $x \phi$ into a positive radial function and a $g_{1}$-integrable function) yield existence of a complete metric on $S$ with $\operatorname{Ric}(g)=R$. 


\section{References}

[CV] S. Cohn-Vossen, Kürzeste Wege und Totalkrümmung auf Flächen, Compositio Math., 2 (1935) 69-133.

[D1] D. DETURCK, Metrics with prescribed Ricci curvature, Ann. of Math. Study 102 (Seminar on Differential Geometry, S. T. Yau, ed., 1982) 525-537.

[F] R. FINN, On a class of conformal metrics, with application to differential geometry in the large, Comment. Math. Helv., 40 (1965) 1-30.

[Ho] L. HÖRMANDER, Linear Partial Differential Operators, Springer-Verlag, 1963.

[H1] A. HUBER, On subharmonic functions and differential geometry in the large, Comment. Math. Helv., 32 (1957) 13-72.

[H2] - Vollständige konforme Metriken und isolierte Singularitäten subharmonischer Funktionen, Comment. Math. Helv., 41 (1966) 105-136.

[HT] D. Hulin and M. TROYANOV, Prescribing curvature on open surfaces, preprint, 1990.

[M] J. MILNOR, On deciding whether a surface is parabolic or hyperbolic, Amer. Math. Monthly, 84 (1977) 43-46.

[PW] M. PROTTER and H. WEINBERGER, Maximum Principles in Differential Equations, Springer-Verlag, 1984.

[R] Y. RESCHETNJACK, Isothermal coordinates on manifolds with bounded curvature, Sibirskii Math. J., 1 (1960) 248-276 (in Russian).

Department of Mathematics,

Cornell University,

Ithaca, NY 14853 U.S. A.

Department of Mathematics,

University of Pennsylvania,

Philadelphia, PA 19104 U. S. A. 